\title{
Root Neighborhoods of a Polynomial
}

\author{
By Ronald G. Mosier
}

\begin{abstract}
The root neighborhoods of $p(z)$, a polynomial over the complex field, are the sets of complex numbers that are the roots of polynomials which are near to $p(z)$. The term 'near' means that the coefficients of the polynomials are within some fixed magnitude of the coefficients of $p(z)$. A necessary and sufficient condition for a complex number to be in the root neighborhoods is given and it is proved that each root neighborhood contains at least one root of $p(z)$ and the same number of roots of each near polynomial. Finally, a necessary and sufficient condition is given for a root neighborhood to contain more than one root of $p(z)$, and consequently more than one root of any of the near polynomials.
\end{abstract}

1. Introduction. In solving polynomial equations, by computer or otherwise, the values of the coefficients are usually rounded off. Much has been done to derive ways to estimate what influence this rounding has on the calculated roots.

The most modern approach is to derive a condition number, a kind of derivative, to estimate the magnitudes of the changes of the roots which correspond to changes in the coefficients. This approach has been very fruitful; the reader is referred to Gautschi [1]-[4] and, of course, the classic works of Wilkinson [9], [10] for further details.

Another approach is to consider the round-off of the coefficients as a continuity problem and to use the geometry of the complex plane. The results obtained are more general and usually weaker than those obtained with the first approach, but provide insight and point out directions of research. The reader is advised to read Ostrowski [7] as an example of this second approach.

This paper is of the second kind and treats polynomial round-off effects as a continuity question. It presents theorems which, though modest, do provide some insight. In addition, the discussion is of an elementary nature and accessible to a wide audience of mathematicians. Finally, the results are useful in the practical work of polynomial root-finding, especially backward error analysis.

2. Preliminaries. Denote by $P_{n}$ the set of polynomials with complex coefficients and degree at most $n$. Let $p(z), q(z) \in P_{n}$, where

$$
p(z)=a_{0}+a_{1} z+\cdots+a_{n} z^{n},
$$

and

$$
q(z)=b_{0}+b_{1} z+\cdots+b_{n} z^{n} .
$$

For a fixed set of positive real numbers, $m_{j}, j=0,1, \ldots, n$, define the metric

$$
d(p, q)=\max \left|a_{j}-b_{j}\right| / m_{j} .
$$

Received August 7, 1985; revised October 18, 1985.

1980 Mathematics Subject Classification. Primary 65G05, 30C15, 30C10; Secondary 12D10, 26C10. 
The $m_{j}$ are weights which determine the relative importance of the variations in each coefficient. If $m_{j}=1.0$ for all $j$, then we are considering absolute differences in the coefficients. If we have some fixed $p(z)$ in mind, then by setting $m_{j}=\left|a_{j}\right|$ for each $j$, we can consider relative differences with respect to the coefficients of $p(z)$. For round-off analysis on a computer, the weights are usually powers of two.

In the practical work of solving polynomial equations, every coefficient is subject to possible round-off, but in the literature it is common to study cases where only a subset of the coefficients, or even just one coefficient, is allowed to be perturbed. In these cases, the metric must be redefined to allow the weights to be nonnegative with the convention that $u / 0=\infty$ for $u>0$, and $0 / 0=0$. In other words, for $m_{j}=0$, $d(p, q)=\infty$, unless $a_{j}=b_{j}$. In order to simplify notation and to avoid the tedium of mentioning the special case of the origin, zero-valued weights will be used in none of the theorems and in only one example, Wilkinson's polynomial. The proofs will assume that $m_{j}>0, j=0,1, \ldots, n$, but the theorems are still true in the case of the extended metric.

With the metric, we can now define neighborhoods of polynomials. For $\varepsilon \geqslant 0$ and $p(z) \in P_{n}$, denote by $N(p, \varepsilon)$ the polynomiais that form a closed ball around $p(z)$ of radius $\varepsilon$ :

$$
N(p, \varepsilon)=\left\{q \in P_{n}: d(p, q) \leqslant \varepsilon\right\} .
$$

Define the set of all roots of the polynomials in $N(p, \varepsilon)$ to be the set $Z(p, \varepsilon)$,

$$
Z(p, \varepsilon)=\{z: q(z)=0 \text { for some } q(z) \in N(p, \varepsilon)\}
$$

The connected components of $Z(p, \varepsilon)$ are the root neighborhoods mentioned in the title of this paper.

3. The Set $Z(p, \varepsilon)$. For a given $p(z) \in P_{n}$, denote by $g(z)$ the function

$$
g(z)=p(z) /\left(m_{0}+m_{1}|z|+\cdots+m_{n}|z|^{n}\right) \text {. }
$$

Occasionally, in order to indicate what polynomial, $p(z)$, was used in forming $g(z)$, we shall write $g(p ; z)$ for $g(z)$.

For any complex value, $u=r e^{i \theta} \neq 0$, denote by $p_{u}(z)$ the polynomial

$$
p_{u}(z)=p(z)-g(u) \sum_{j=0}^{n} m_{j} e^{-i j \theta} z^{j}
$$

with the special case

$$
p_{0}(z)=p(z)-m_{0} g(0)=p(z)-a_{0} .
$$

LEMMA. Using the definitions and the notation above, we have that

(i) $p_{u}(u)=0$

(ii) $p_{u}(z) \in N(p,|g(u)|)$, and

(iii) if $q(z) \in P_{n}$ and $q(u)=0$, then $d(p, q) \geqslant d\left(p, p_{u}\right)$.

Proof. Both (i) and (ii) are obvious. For (iii) note that

$$
d(p, q) \sum_{j=0}^{n} m_{j}|z|^{j} \geqslant|p(z)-q(z)| .
$$

So, since $q(u)=0$, we have that

$$
d(p, q) \sum_{j=0}^{n} m_{j}|u|^{j} \geqslant|p(u)| .
$$


Dividing by the summation term gives

$$
d(p, q) \geqslant|g(u)|=d\left(p, p_{u}\right),
$$

which completes the proof.

The first theorem identifies the set $Z(p, \varepsilon)$.

Theorem 1. Given $p(z) \in P_{n}$, then

$$
Z(p, \varepsilon)=\{u:|g(p ; u)| \leqslant \varepsilon\} .
$$

Proof. If $|g(u)| \leqslant \varepsilon$, then from the Lemma, $p_{u}(z) \in N(p, \varepsilon)$ and has $u$ as a root, so $u \in Z(p, \varepsilon)$.

On the other hand, if $q(z) \in N(p, \varepsilon)$ and $q(u)=0$, then also from the Lemma,

$$
|g(u)|=d\left(p, p_{u}\right) \leqslant d(p, q) \leqslant \varepsilon,
$$

which proves the theorem.

Figure 1 shows $Z(p, 0.01), Z(p, 0.008)$, and $Z(p, 0.004)$ for $p(z)=z^{2}-$ $(10.5+i 10.2) z+(1.5+i 53.5)$, which has roots at $5+i 5$ and $5.5+i 5.2$. The weights used were $m_{0}=4, m_{1}=0.5$, and $m_{2}=0.01$.

A second example is Figure 2, which shows a part of $Z\left(w, 2^{-23}\right)$ for what we shall call Wilkinson's polynomial

$$
w(z)=(z-1)(z-2) \cdots(z-20) .
$$

The weights used were the ones Wilkinson [9] used, $m_{19}=1$ and $m_{j}=0$ for $j \neq 19$. The reader is referred to the remark following the definition of the metric in Section 2.

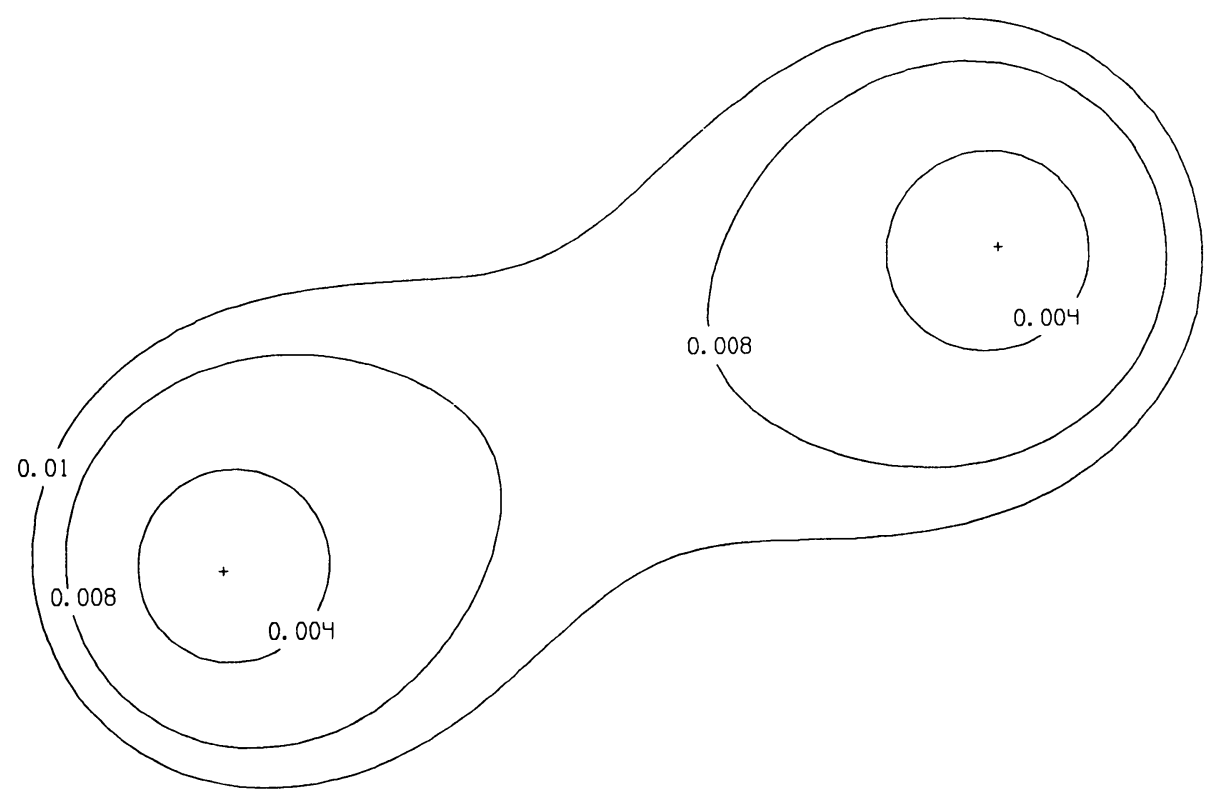

FIGURE 1 


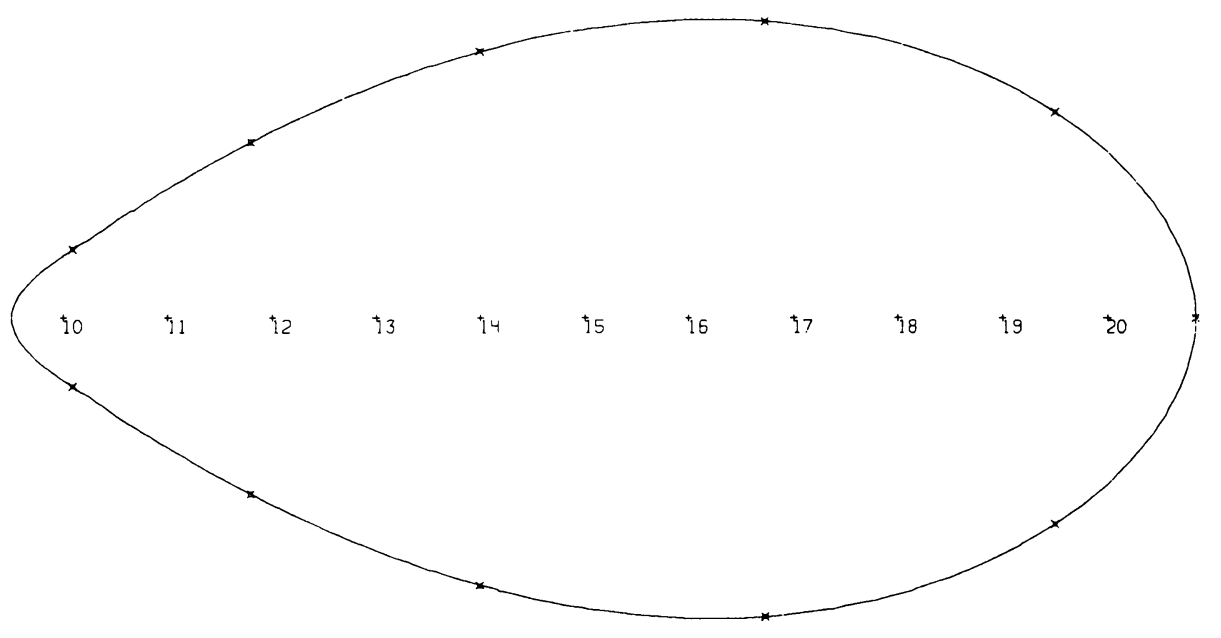

+ ROOTS OF $W(Z)$

$\times$ ROOTS OF PERTURBED $W(Z)$

Figure 2

The roots of $w(z)$ which are visible in this piece of the complex plane are marked in Figure 2, as are the roots of the perturbed polynomial $w(z)-2^{-23} z^{19}$. Notice that for the chosen weight configuration, the perturbed polynomial is in fact $w_{u}(z)$, where $u$ is one of its real roots.

To show these facts and to show how useful Theorem 1 is for backward error analysis, observe Table 1, which shows the roots of the perturbed polynomial to nine decimal places and $\log _{2}|g(w ; u)|$ to four decimal places for each computed root. The roots not listed in the table agree up to nine decimal places to the exact roots of $w(z)$.

\section{TABLE 1}

Backward error analysis for Wilkinson's polynomial $w(z)$

Computed root $u$

20.846908101

19. $502439400+i 1.940330347$

$16.730737466+i 2.812624894$

$13.992358137+i 2.518830070$

$11.793633881+i 1.652329728$

$10.095266145+i 0.643500904$

8.917250249

8.007267603

6.999697234

6.000006944

4.999999928

\section{$\underline{\log _{2}|g(w ; u)|}$}

$-23.0000$

$-23.0000$

$-23.0000$

$-23.0000$

$-23.0000$

$-23.0000$

$-23.0000$

$-23.0000$

$-23.0000$

$-23.0000$

$-23.0090$ 
From Theorem 1, we may conclude that for any $u \in Z(p, \varepsilon)$ there is at least one root, say $v$, of $p(z)$ so that

$$
|v-u| \leqslant \sqrt[n]{\left(\varepsilon /\left|a_{n}\right|\right) \sum_{j=0}^{n} m_{j}|u|^{j}},
$$

and also there is at least one root, say $y$, so that

$$
|1-y / u| \leqslant \sqrt[n]{\left(\varepsilon /\left|a_{n}\right|\right) \sum_{j=0}^{n} m_{j} /|u|^{n-j}} .
$$

Because of the $1 / n$ exponent, these bounds are very weak for small $\varepsilon$, especially compared to bounds found using the condition number approach, but they are also very general and are intended to illustrate how the magnitudes of the roots determine the absolute and relative errors possible. Wilkinson first pointed out that large roots are subject to larger displacements by perturbations of the coefficients and that smaller roots have larger relative errors. Formulas (3.1) and (3.2) suggest this observation. See Wilkinson [9].

4. Root Neighborhoods of $p$. As $\varepsilon$ increases, the set $Z(p, \varepsilon)$ increases; for $\varepsilon$ large enough $Z(p, \varepsilon)$ is no longer bounded. As an example, $p(z)=\sum m_{j} z^{j}$ has $g(p ; r)=$ 1 for every nonnegative real number $r$. For the remainder of this paper we will consider only $\varepsilon<\left|a_{n}\right| / m_{n}$. This is no great restriction, since in the sense of round-off, it implies that we are sure of at least the degree of the polynomial. It is a great advantage, however, because it allows us to conclude that $Z(p, \varepsilon)$ is bounded.

THEOREM 2. For any $0<\varepsilon<\left|a_{n}\right| / m_{n}$, if $q(z) \in N(p, \varepsilon)$, then $q(z)$ and $p(z)$ have the same number of roots, counting multiplicities, in each connected component of $Z(p, \varepsilon)$. Furthermore, there is at least one root of $p(z)$ in each connected component of $Z(p, \varepsilon)$.

Proof. Since $\left|a_{n}\right| / m_{n}>0, p(z)$ has $n$ roots, counting multiplicities. Since $\left|a_{n}\right| / m_{n}$ $>\varepsilon, q(z)$ has $n$ roots, counting multiplicities, as does every member of the family of polynomials,

$$
f_{\lambda}(z)=\lambda p(z)+(1-\lambda) q(z), \quad 0 \leqslant \lambda \leqslant 1
$$

Furthermore, since

$$
d\left(f_{\lambda}, p\right)=(1-\lambda) d(q, p) \leqslant \varepsilon, \quad 0 \leqslant \lambda \leqslant 1,
$$

we have that all the roots of $f_{\lambda}(z), 0 \leqslant \lambda \leqslant 1$, lie in $Z(p, \varepsilon)$ by definition.

The coefficients of $f_{\lambda}(z)$ are linear functions of $\lambda$, and it is well known that the roots of a polynomial are continuous functions of the coefficients. Hence, as $\lambda$ varies from 0 to 1 the roots of $f_{\lambda}(z)$ trace continuous paths from the roots of $f_{0}(z)$ to the roots of $f_{1}(z)$.

Let $f_{0}(z)$ have $k$ roots in some connected component of $Z(p, \varepsilon)$, the remaining 
$n-k$ roots being isolated in the other connected components. It follows that this will hold true for the roots of all $f_{\lambda}(z)$ for $\lambda$ up to and including 1 . Since $f_{0}(z)=q(z)$, and $f_{1}(z)=p(z)$, the first assertion is proved.

The second assertion follows immediately from the first and the definition of $Z(p, \varepsilon)$.

The fact that each connected component of $Z(p, \varepsilon)$ contains at least one root of $p(z)$ and a corresponding root of $q(z)$ for any $q(z) \in N(p, \varepsilon)$, justifies our calling the connected components of the set $Z(p, \varepsilon)$, the root neighborhoods of $p$.

The paths which are traced by the roots of the polynomials

$$
\lambda p(z)+(1-\lambda) p_{u}(z), \quad 0 \leqslant \lambda
$$

are easy to follow. They are the curves

$$
\arg \left(p(z) / \sum_{k=0}^{n} m_{k} e^{-i k \theta} z^{k}\right)=\arg g(u),
$$

where $\theta$ is the argument of $u$.

As an example, we have drawn the root paths for the polynomial family

$$
w(z)-\lambda 2^{-23} z^{19}, \quad 0 \leqslant \lambda,
$$

in Figure 3. The roots trace the curves $\arg \left(w(z) / z^{19}\right)=0$; they pass through the roots of Wilkinson's perturbed polynomial and continue through to the roots of $w(z)$. The perturbed roots are marked by asterisks, as they were in Figure 2, and the roots of $w(z)$ are labeled. The real axis has not been drawn; what appears to be the real axis are parts of the root paths. The paths beginning at complex pairs collide on the real axis at the points marked by the diamond-shaped symbols and then split apart and follow the real axis to the roots of $w(z)$. These singularities on the root paths are called collision points in the literature (see Gautschi [4]).

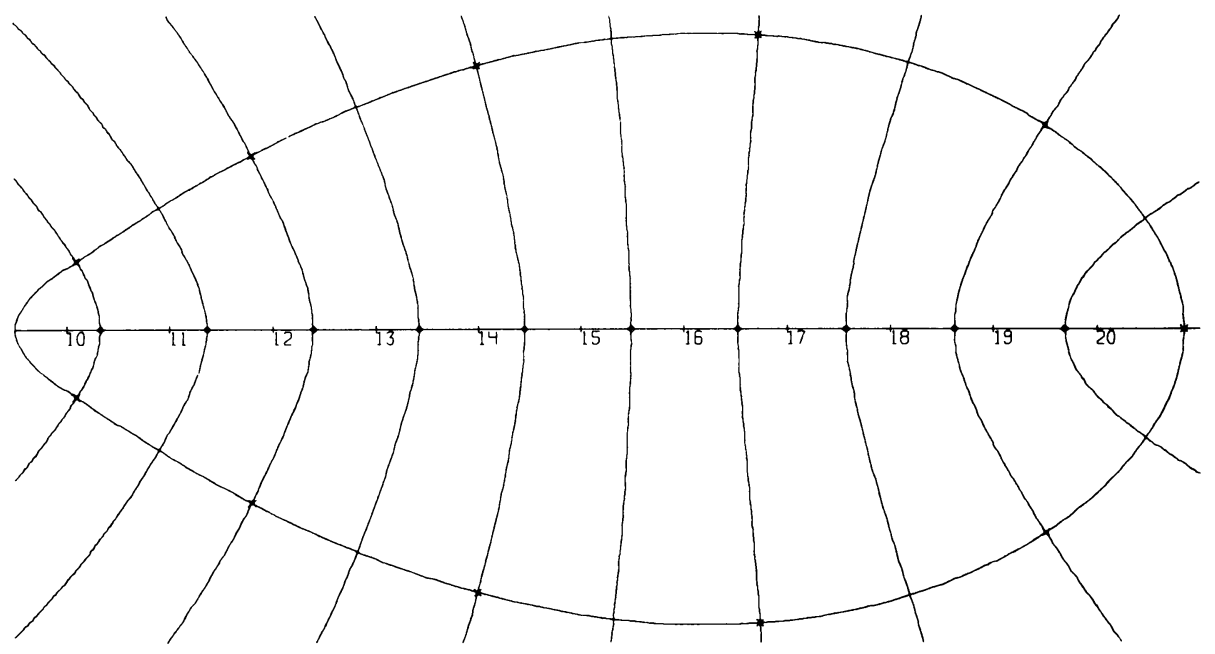

Figure 3 
Figure 3 also shows the root paths of the family of polynomials,

$$
w(z)+\lambda 2^{-23} z^{19}, \quad 0 \leqslant \lambda,
$$

which are the curves $\arg \left(w(z) / z^{19}\right)=\pi$. These are the paths in Figure 3 which pass through the unmarked points of the boundary of the root neighborhood. Again, note the collision points on the real axis.

The next theorem establishes the importance of the collision points and clarifies what they are.

THEOREM 3. A root neighborhood of $p$ contains two roots of $p(z)$ if and only if it contains a u such that $p_{u}(z)$ has a double root at $u$.

Proof. Clearly, from Theorem 2, if $p_{u}(z)$ has a double root at $u$ then $p(z)$ must have two roots in the root neighborhood containing $u$.

To prove the converse, let $z_{1}$ and $z_{2}$ be two distinct roots of $p(z)$ and suppose that both lie in the same root neighborhood of $Z(p, \varepsilon)$.

Define

$$
\delta=\inf \left\{\lambda: z_{1} \text { and } z_{2} \text { lie in the same root nbd of } Z(p, \lambda)\right\} .
$$

From this definition, we can say that $z_{1}$ and $z_{2}$ lie in the same root neighborhood of $Z(p, \delta)$, that neighborhood being the limit of a decreasing family of compact and connected sets.

It is straightforward to show, using the continuity of the roots of a polynomial with respect to the coefficients and the definition of $\delta$, that the root neighborhood of $Z(p, \delta)$ which contains both $z_{1}$ and $z_{2}$ is composed of two sets, one containing $z_{1}$ and the other containing $z_{2}$. Further, these sets have disjoint interiors and intersecting boundaries. This says that the curve enclosing the root neighborhood either crosses itself or is tangent to itself at some point $u$.

Considering the boundary of the root neighborhood as a function of two variables, $|g(r, \theta)|=\delta$, where $z=r e^{i \theta}$, we know that if $u \neq 0$ we have at $u$,

$$
\partial|g| / \partial r=0 \quad \text { and } \quad \partial|g| / \partial \theta=0
$$

An exercise in differentiation shows that

$$
\partial|g| / \partial r=\operatorname{Re}(\partial g / \partial r \bar{g} /|g|)
$$

and that

$$
\partial|g| / \partial \theta=-\operatorname{Im}(r \partial g / \partial r \bar{g} /|g|)
$$

Therefore, at the nonzero point $u$,

$$
\partial g / \partial r=0
$$

However, from the definition of $p_{u}(z)$, one can easily prove that $\partial g / \partial r=0$ at $u$ when and only when $u$ is a double root of $p_{u}(z)$, proving the theorem for $u \neq 0$.

Assume $u=0$ is a simple root of $p_{0}(z)$ and the only point where the boundary $|g(r, \theta)|=\delta$ crosses itself. We argue as follows: 
Let $F_{1}$ and $F_{2}$ be the sets making up the root neighborhood with $z_{1} \in F_{1}$ and with $z_{2} \in F_{2}$, and suppose that in addition to zero, $p_{0}(z)$ has $k_{1}$ roots in $F_{1}$ and $k_{2}$ roots in $F_{2}$.

Choose some $x$ in the interior of $F_{1}$, but with $d\left(p_{x}, p_{0}\right)$ small enough so that by the continuity of the roots of a polynomial with respect to the coefficients, $p_{x}(z)$ has $k_{1}$ roots in $F_{1}$ near the roots of $p_{0}(z)$ and $k_{2}$ roots in $F_{2}$ near the roots of $p_{0}(z)$ which are there. Of course, it has one more root in $F_{1}, x$, which is close to zero.

Do the same thing for some $y$ in the interior of $F_{2}$, so that $p_{y}(z)$ has $k_{1}$ roots in $F_{1}$ and $k_{2}+1$ roots in $F_{2}$, including $y$.

Then $p_{x}(z)$ and $p_{y}(z)$ do not have the same number of roots in two root neighborhoods of $Z(p, \max \{|g(x)|,|g(y)|\})$, contradicting Theorem 2.

We conclude that the assumption is wrong; either $p_{0}(z)$ does have other roots common to both boundaries, or zero is a double root of $p_{0}(z)$. In either case the theorem is proved.

As an illustration of the $Z(p, \delta)$ of Theorem 3, Figure 4 shows, for the polynomial of Figure 1, two small root neighborhoods which separate the roots, a large root neighborhood which contains both roots, and the root neighborhood whose boundary contains a point $u$ such that $p_{u}(z)$ has a double root at $u$. This root neighborhood just manages to contain both roots. The point $u$ is obvious in the center of the drawing.

According to the proof of Theorem 3, the collision points of Figure 2, occur when $\partial g / \partial r=0$. As an application, we state an easy consequence for polynomials with real roots.

Let $p(x)$ have only simple real roots; then on the real axis $|g(x)|$ takes on $n-1$ local maxima. Suppose that the local maxima that are $\leqslant \varepsilon$ are $k$ in number and are in $j$ root neighborhoods. Then any $q(x) \in N(p, \varepsilon)$ with real coefficients has at most $(k+j) / 2$ complex pairs as roots.

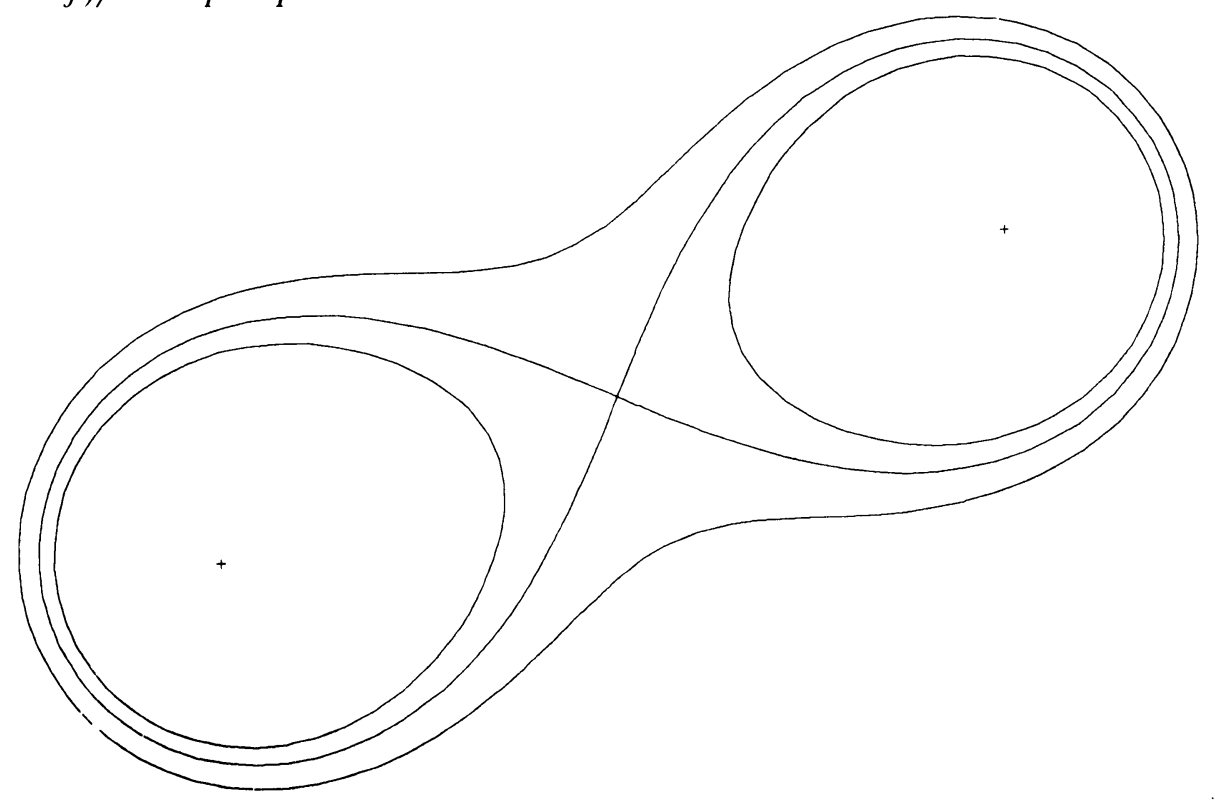

FIGURE 4 
Table 2 shows the local maxima for $w(z)$. The perturbed polynomial has exactly five complex pairs which agrees exactly with the table. Figure 3 also agrees with the table.

TABLE 2

\begin{tabular}{|c|c|}
\hline location of maximum & $\log _{2}|g|$ \\
\hline 1.045893 & 50.8425 \\
\hline 2.084519 & 28.4906 \\
\hline 3.119416 & 14.7239 \\
\hline 4.151980 & 4.7060 \\
\hline 5.182987 & -3.1165 \\
\hline 6.212943 & -9.4502 \\
\hline 7.242221 & -14.677 \\
\hline 8.271119 & -19.029 \\
\hline 9.299900 & -22.652 \\
\hline 10.328817 & -25.646 \\
\hline 11.358132 & -28.077 \\
\hline 12.388138 & -29.988 \\
\hline 13.419191 & -31.406 \\
\hline 14.451759 & -32.340 \\
\hline 15.486500 & -32.785 \\
\hline 16.524440 & -32.712 \\
\hline 17.567379 & -32.060 \\
\hline 18.619104 & -30.706 \\
\hline 19.690939 & -28.342 \\
\hline
\end{tabular}

Department of Applied Mathematics

CIMS 418-11-44

Chrysler Corporation

Box 1118

Detroit, Michigan 48288

1. W. Gautschi, "The condition of orthogonal polynomials," Math. Comp.., v. 26, 1972, pp. 923-924.

2. W. Gautschi, "On the condition of algebraic equations," Numer. Math., v. 21, 1973, pp. 405-424.

3. W. GautsChI, "The condition of polynomials in power form," Math. Comp., v. 33, 1979, pp. $343-352$.

4. W. Gautschi, "Questions of numerical condition related to polynomials," MAA Studies in Numerical Analysis, Vol. 24 (Gene H. Golub, ed.), The Mathematical Association of America, 1984, pp. 14()-177.

5. K. Kuratowski, Topologi, Vol. II, Academic Press, New York and London, 1968.

6. M. Marden, Geometry of Polynomials, 2nd ed., Math. Surveys, No. 3, Amer. Math. Soc., Providence, R.I., 1966.

7. A. M. Ostrowski, Solutions of Equations and Systems of Equations, Academic Press, New York and London, 1960.

8. S. SAKs \& A. ZYGmund, Analytic Functions, Elsevier, New York, 1971.

9. J. H. Wilkinson, Rounding Errors in Algebraic Processes, Prentice-Hall, Englewood Cliffs, N.J., 1963.

10. J. H. Wilkinson, The Algebraic Eigenvalue Problem, Claredon Press, Oxford, 1965. 